\title{
Design and Simulation for Bionic Mechanical Arm in Jujube Transplanter*
}

\author{
Yonghua Sun, Wei Wang ${ }^{* *}$, Wangyuan Zong, and Hong Zhang \\ Zibo in Shandong, Lecturer, Mechatronics \\ Tel.: 0997-4683859 13031270332 \\ sunyoghua@sina.cn
}

\begin{abstract}
In this paper an automatic bionic mechanical arm of jujube transplanter has been designed and simulated with Pro/E and ADAMS software. The device can achieve the work of clamping — sending — setting the sapling and support the sapling to guarantee it perpendicularity in setting process. Design the structure of manipulator utilizing the simulation of hand working. There is 5-DOF at the manipulator to achieve simulating. Constitute dynamics mathematical model and estimated inseminate error of bionic manipulator. The three dimensional model of the manipulator was build up and simulated by using Pro/E software. Then up build the virtual prototype and kinetics simulation in ADAMS software and chalk up the dynamics parameter curve of clamping force etc. This manipulator will establish theory and practice foundation to the cyber-identify and cyber-supervise of sapling translating.
\end{abstract}

\section{Introduction}

Mechanical arm have been designed for jujube transplanter aiming at the outside diameter and characteristic of tree form, to meet new requirement in south Xinjiang, featured with new planting mode that called lower stem and high-density. It is designed by simulating the supporting mechanism of human hand. This new design can improve planting stability and planting efficiency, under the help of the support from mechanical arm. The mechanism is the core component of the transplanter that directly affects the quality of seedlings planted.

\section{Working Principle and Structure}

The bionic mechanical arm is composed by structures of upper and lower splint $(5,7)$, link (4), spring (3), roller $(2,8)$ and link (1) and pin etc. There is five-freedom except the rollers that can let the manipulator realize the opening, closing, turning as well as other operations.

\footnotetext{
* Project Funding: Subsidized by Sinkiang Science and Technology Supporting Projects (2009zj19)

** Corresponding author.
} 


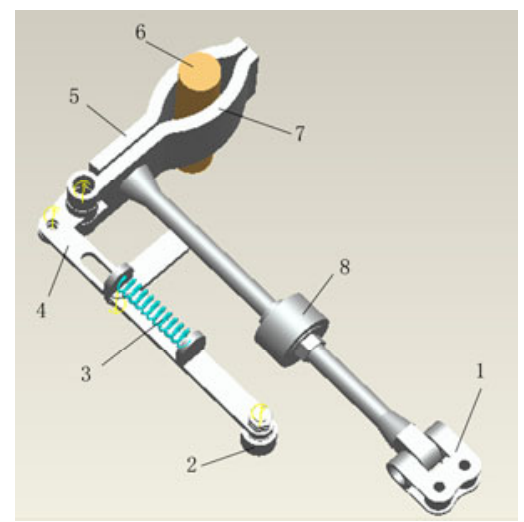

Fig. 1. Structure Diagram of Bionic Mechanical arm

1. Chain-Element 2.First Roller 3.Spring 4.Link 5.Upper Splint 6.Sapling 7.Lower Splint 8.Second Roller

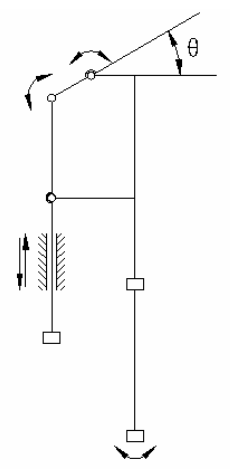

Fig. 2. Schematic Diagram of Bionic Mechanical Arm

In working, chain-element (1) link to the driving chain and moving at a certain speed. The first roller (2) and second roller (8) run in each orbit. The manipulator is supported by the second roller (8). The first roller (2) able to automatic control the opening and closing of upper splint (6) is located at the obit with variable size and fitted to spring (3). Figure 1 is location of spring at the state of compression and now the manipulator will keep current state for a minute until completed sapling planted. Then the upper splint (6) will detach the sapling with opening angle $\theta$ by spring force as the change in size of guide and wait for the next operation. Figure 2 shows the working principle. The opening angle $\theta$ of hand is controlled by a long slot between guide and strut. Parallel mobile is main movement of link (4) which attach to the relevant parts of lower splint by hinge pin. 


\section{Motion Mathematical Models}

There will be a little offset error to sapling`s right location in actual planting process because of linker in structure according to the working principle of the bionic mechanical arm. Hypothesis the diameter of sapling be handed is $d$, and the distance that link moving down is $h$, now, the dip of upper splint is $\theta$. Ideally, the field angle is $\theta$ between the two hand splint if there is no movement of lower splint. Ideally, the field angle between two splints is $\beta=\theta-\alpha$ If considering the dip $\alpha$ of lower splint as the structure factor. That is the error angle is $\beta=\theta-\alpha$.

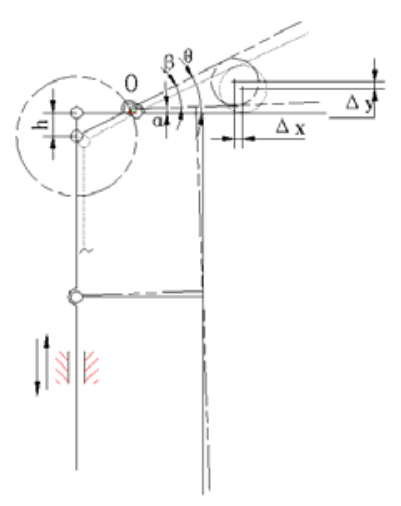

Fig. 3. Working Locus of Manipulator

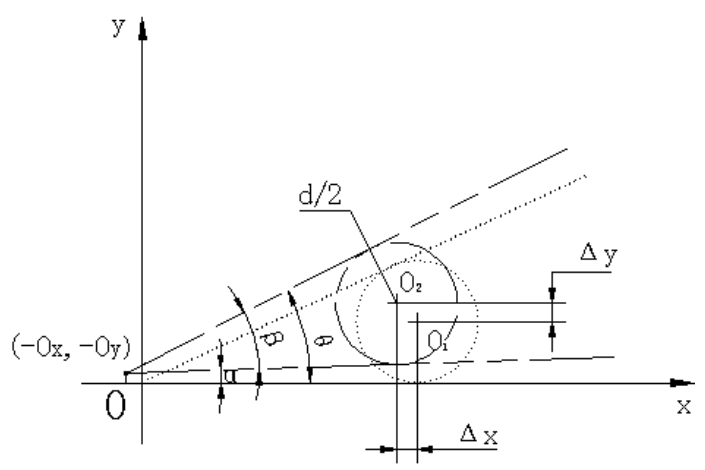

Fig. 4. Error Coordinates in Sapling Planted of Manipulator

Define the original point $o$ as the coordinate origin to analysis the offset of sapling planting. Establish the coordinates shown in Figure 4. The coordinate sapling planted is $(x 1, y 1)$ in ideally and the actual coordinate is $(x 2, y 2)$ after considering the coordinate error if hypothesis the original point as $(-O x,-O y)$ after migrated. We can get the follow mathematic model according to its trajectory. 
The coordinate sapling planted in ideally:

$\left\{\begin{array}{l}x 1=d \times \cot \frac{\beta}{2} \\ y 1=d\end{array}\right.$

The actual coordinate:

$$
\left\{\begin{array}{l}
x 2=\frac{d \cos (\alpha+\beta / 2)}{\sin (\beta / 2)}-O x \\
y 2=\frac{d \sin (\alpha+\beta / 2)}{\sin (\beta / 2)}+O y
\end{array}\right.
$$

The mathematic model of sapling planted error in actually:

$$
\left\{\begin{array}{l}
\Delta x=-\left(x_{2}-x_{1}\right)=d \cot (\beta / 2)-\frac{d \cos (\alpha+\beta / 2)}{\sin (\beta / 2)}+O x \\
\Delta y=y_{2}-y_{1}=\frac{d \sin (\alpha+\beta / 2)}{\sin (\beta / 2)}-d+O y
\end{array}\right.
$$

$\Delta \mathrm{x}, \Delta \mathrm{y}$ were sapling planted error in $\mathrm{x}, \mathrm{y}$ direction.

\section{Dynamic Simulation Analysis}

\subsection{Virtual Prototype Design Based on Adams}

Figure 5 shows the virtual prototype designed in ADAMS which comes from the three-dimensional model of bionic mechanical arm established in PROE. Set up the

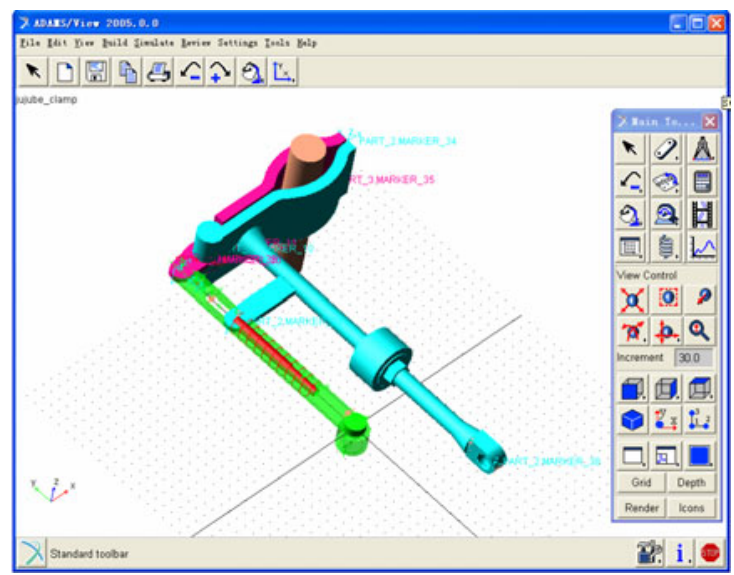

Fig. 5. Modeling of Virtual Prototype 


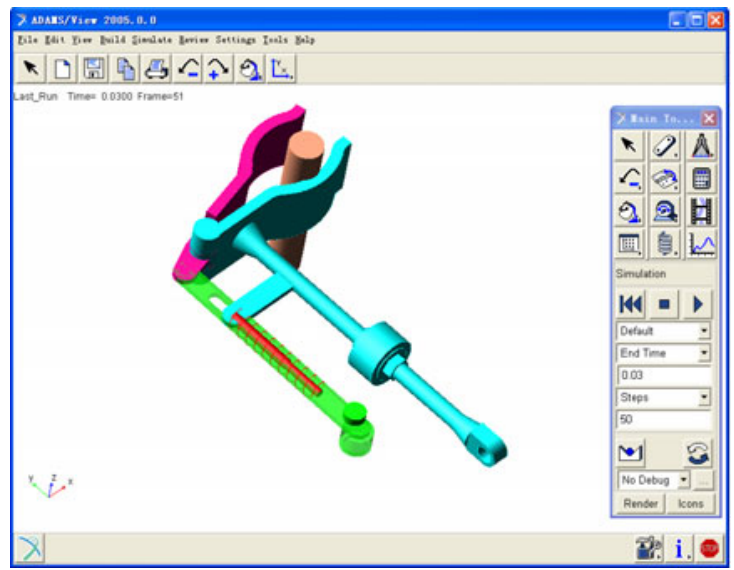

Fig. 6. Dynamic Simulation of Virtual Prototype

attribute as materials of various parts as stainless unity and so on. Set the connect sets between the parts and load the property of spring such elastic stiffness coefficient as $K=800$ and the damping coefficient as $C=0.5$. Then to exert the force $F$ in the connecting rod end and set the running time as $0.03 \mathrm{~s}$ and the step as 50 , and then operate it to perform the dynamic simulation for the prototype. Afterward, we will obtain a motion state diagram likes Figure 6.

\subsection{Kinematics and Dynamic Simulation Analysis}

Change the size of the force $F$ in connecting rod end at the same kinematics time and measure the angle caused by compression force on the spring, field angle of the upper and down hand splint and sapling planted position error. Then get the variation curve of various spring force $F$, field angle- 1 and error angle- 2 respectively in the time of $t=0.03 \mathrm{~s}$. Follow the Figure 7, Figure 8, Figure 9.

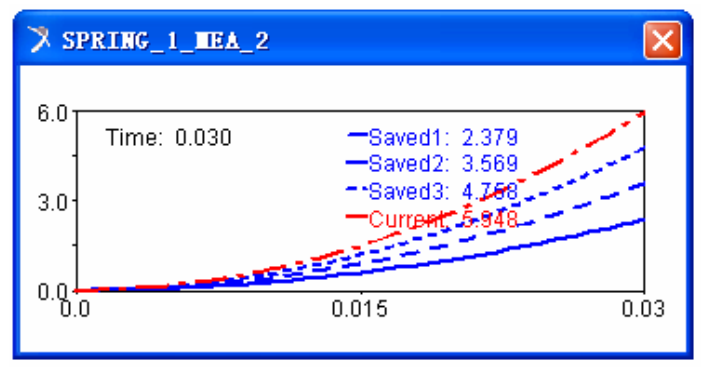

Fig. 7. Variation Curve of Spring Force in Time on Different Force $F$ 


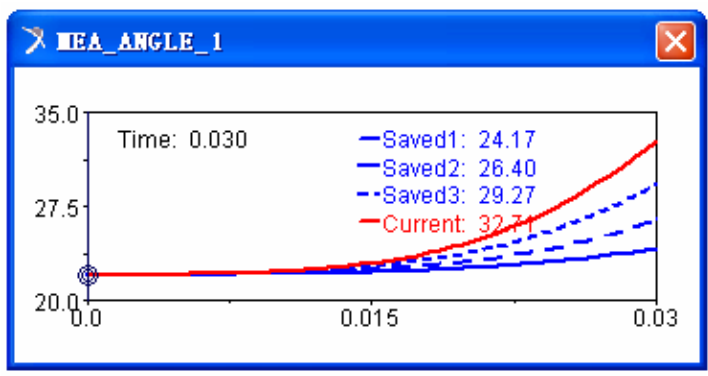

Fig. 8. Variation Curve of Field Angle of Plant Holder`s Hand on Different Force $F$

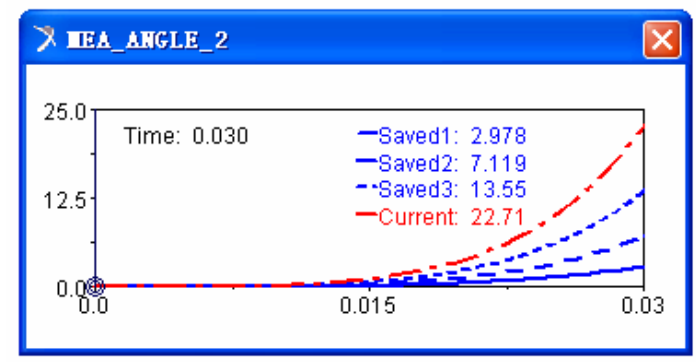

Fig. 9. Variation Curve of Planted Error on Different Force $F$

Select the preloading as $0.2 \sim 0.3 \mathrm{~N}$ according to the curve analysis of each parameter mentioned above and its operating characteristics and requirements. Now the field angle can meet the demand for planting seedlings and have a little error. Therefore, the hand field angle $30^{\circ}$ able to be the limit position to improve the relative motion quantity of the link.

Analysis the position change, velocity and the acceleration of the load component force in $X$ direction at the time that exert the force $0.3 \mathrm{~N}$ in connection rot as the preload force and get the variation curve as Figure 10. The load component force is more stable in the intermediate section. Analysis the elastic, change speed and amount of compression of compression spring and get the variation curve as Figure 11.

Table 1. Kinetic Parameters in Prescriptive Time on Different Force

\begin{tabular}{|c|r|r|r|}
\hline $\begin{array}{l}\text { Kanetic } \\
\text { Prameters } \\
\text { Preloading (N) }\end{array}$ & $\begin{array}{l}\mathrm{F}(\text { spring) } \\
(\mathrm{N})\end{array}$ & $\begin{array}{l}\text { Angle-1 } \\
\left({ }^{\circ}\right)\end{array}$ & $\begin{array}{l}\text { Angle-2 } \\
\left({ }^{\circ}\right)\end{array}$ \\
\hline 0.1 & 5.948 & 32.71 & 22.71 \\
\hline 0.2 & 4.758 & 29.27 & 13.55 \\
\hline 0.3 & 3.569 & 26.40 & 7.119 \\
\hline 0.4 & 2.379 & 24.17 & 2.978 \\
\hline
\end{tabular}




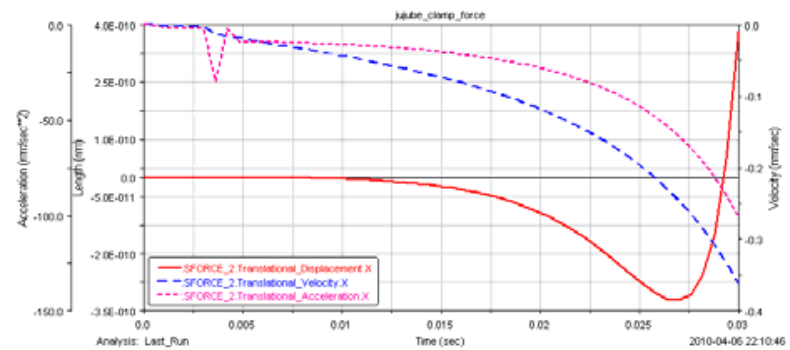

Fig. 10. Variation Curve of Preload in X Direction

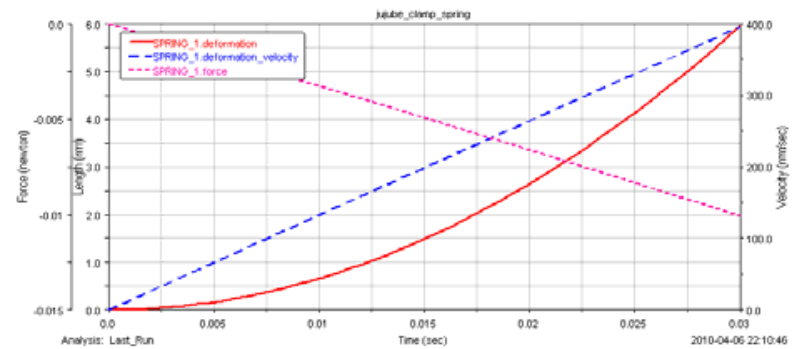

Fig. 11. Variation Curve of Elastic of Compression Spring

\section{Conclusions}

In this paper, automatic mechanical arm which imitates human hand to support sapling is designed by using new operation principle of the manipulator. Analysis and establish mathematical model of the planted error to meet requirement of the jujube transplanting demand for lower-density inseminate mode. Design the corresponding structure of the manipulator use of above mathematical model. Build modeling in PROE, introduce it into ADAMS and establish virtual prototype in it. Do the simulation experiment for primary related parameters of the prototype and draw the corresponding variation curve. The clamping force to sapling is not much according to the curve analysis of each dynamic parameter of the virtual prototype of planted manipulator, so it can realize base on the elastic of spring. Furthermore, clamping force can be adjustable according to the diameter of the sapling, with the adaptive ability of spring during the working process.

\section{References}

[1] Zhang, M., Li, S.: Optimum Planting Depth to Poplar Mechanical Afforestation in Subarid Sand, vol. (4), pp. 4-5. Jilin Forestry Science and Technology, Changchun (2004)

[2] Dong, Q., Han, L.: Cultivation Techniques for High Yield of Jujube in Sandy. Inner Mongolia Forestry Investigation and Design, vol. (4), pp. 83-84. Forestry Survey \& Design Institute, Hohhot (2008) 
[3] Li , J., Xiao, H., Hu, Z.: Kinematics Simulation of Mechanical Arm Based on ADAMS. Machine Tool \& Hydraulics (8), 206-209 (2009)

[4] Feng, S., Xie, J., Zhu, W., Ma L.Z.: The Motion Control Study of The Automatic Transplanting Robot. Machinery Design \& Manufacture (3), 166-168 (2008) 